\title{
Die verfassungspolitische Diskussion über die Einrichtung Österreichs als Bundesstaat*
}

Meine Aufgabe ist keine ganz leichte. Ich soll eine Geschichte erzählen, die Sie alle schon kennen, die schon tausende Male erzählt worden ist und die in vielen Versionen kursiert. Im Grunde handelt es sich um die Geschichte der österreichischen Verfassung. Das birgt die Gefahr, den Gründungslegenden, die in reicher Zahl kursieren, eine weitere hinzuzufügen. Um sie möglichst zu bannen, habe ich einen chronologischen Zugang gewählt und den Versuch unternommen, die verfassungspolitische Diskussion um die Einrichtung Österreichs als Bundesstaat in einzelne Phasen zu unterteilen; und im Zuge der Arbeit hat sich gezeigt, dass eine solche Periodisierung ihre innere Berechtigung hat. Wenn ich im folgenden Beitrag vorschlage, die Zeit zwischen Oktober 1918 und November 1920 in sechs Phasen zu gliedern, dann aus der Überzeugung, dass in diesen Perioden jeweils andere Kräfte die Diskussion angetrieben und dass sich Themen wie Einstellungen geändert haben.

\section{Oktober 1918 bis Jänner 1919: Begründung der Republik in den bestehenden Strukturen}

Die erste, von Oktober 1918 bis Jänner 1919 währende Periode ist im Grunde eine Vorphase, gekennzeichnet dadurch, dass es eine Bundesstaatsdiskussion noch nicht gab. Beim Zusammenbruch der Donaumonarchie stellte zunächst niemand die Frage, ob das neue Österreich als Einheitsstaat oder als Bundesstaat eingerichtet werden sollte, denn man war mit anderen Ent- scheidungen mehr als ausgelastet. ${ }^{1}$ Es ging in erster Linie darum, auf dem Boden der einstürzenden Monarchie in ihren deutschstämmigen Gebieten eine Staatsgewalt zu begründen, die sich nicht vom schwarz-gelben Österreich speiste: An Stelle der sich in Abwicklung befindlichen k.k. Ministerien und des in seine Nationalitäten zerfallenden Reichsrats wurden deutschösterreichische Institutionen geschaffen. In zweiter Linie ging es darum, den Kaiser zum Abdanken zu bewegen und eine Republik aufzurichten. Die Sozialdemokraten verfolgten vom ersten Taumeln der Monarchie an das Ziel, an ihre Stelle eine demokratische Republik zu setzen. Nachdem es gelungen war, die anfangs zaudernden Großdeutschen und die über einen starken monarchistischen Flügel verfügenden Christlichsozialen zum Einlenken zu bewegen, ${ }^{2}$

\footnotetext{
* Schriftfassung eines Vortrages, den der Verfasser am 19. 11. 2008 auf der vom Institut für Rechts- und Verfassungsgeschichte der Rechtswissenschaftlichen Fakultät der Universität Wien und vom Österreichischen Staatsarchiv veranstalteten Tagung "Staatsgründung und Verfassungsordnung“ in Wien gehalten hat. Der Verfasser und die Herausgeber danken dem Institutsvorstand, Prof. Thomas Simon, für seine Zustimmung zur Veröffentlichung des Beitrages in dieser Zeitschrift.

${ }^{1}$ Vgl. statt aller die Darstellung bei BRAUNEDER, Deutsch-Österreich 32ff., 79ff., 117ff.

${ }^{2}$ Zur Ausgangssituation und zum Kräfteverhältnis der politischen Lager vgl. GOLDINGER, BINDER, Republik Österreich 13ff.; ABLEITINGER, Grundlegung 148ff.; zu den drei großen Parteien NECK, Sozialdemokratie 227ff.; Staudinger, Christlichsoziale Partei 252ff.; WANDRUSZKA, Nationales Lager 278ff.
} 
wurde dieses Ziel von allen Parteien gemeinsam verfolgt: schrittweise, maßvoll, dem Kaiser gegenüber erstaunlich taktvoll, aber mit sofortiger Absicherung des gewonnenen Terrains im Wege des Rechts. Um die Etablierung neuer zentralistischer oder föderalistischer Strukturen ging es nicht, denn an dieser Nebenfront konnte und wollte niemand kämpfen.

Das Verhältnis zwischen Staat und Ländern verblieb so in den vertrauten Bahnen. Die neue Verfassung war bewusst als Notdach konzipiert, das verhindern sollte, dass das im Übrigen weitgehend unverändert gebliebene Gebäude Schaden nimmt. ${ }^{3}$ Bis Jahresende gelang es, im jungen staatlichen Gebilde die quasiföderalen Strukturen der Donaumonarchie schrittweise zu rekonstruieren. Die Gesetzgebungsbefugnis der Länder wurde anerkannt, ihre Autonomie wurde ausgebaut - aber es blieb eine Autonomie, es war keine Staatlichkeit als Glied eines Bundes.

Diese Bewertung der Vorgänge des Jahres $1918^{4}$ ist keine allgemein akzeptierte. Schon für diese Phase ist ein Gegensatz zwischen zentralistischem Staatsrat und föderalistischen Ländern behauptet und ein politischer Wille zur Veränderung der bestehenden Strukturen registriert worden, bezeichnender Weise in gegenläufige Richtungen. Nach Kelsen, ${ }^{5}$ Merkl $^{6}$ und anderen (nicht immer der Wiener Schule nahestehenden) Juristen, ${ }^{7}$ aber auch nach Auffassung vieler

\footnotetext{
${ }^{3}$ Das Bild stammt von RENNER, Zum Geleite, in: KELSEN, Verfassungsgesetze 1, IV. KELSEN, ebd. VI, spricht demgegenüber von einem "Notbau“.

${ }^{4}$ Sie geht zurück auf und ist breiter dargelegt bei KÖFNER, Revolutionen 134ff.; ABLEITINGER, Grundlegung 151ff.; BRAUNEDER, Gesamtstaat - Länder 30ff.; DERS., Deutsch-Österreich 94ff.

${ }^{5}$ Kelsen, Verfassungsgesetze 1, 18, 103ff.; DERS., Österreichisches Staatsrecht 97ff.

${ }^{6}$ MERKL, Organisation 11; DERS., Verfassung Deutschösterreich 58.

${ }^{7}$ ADAMOVICH, SPANNER, Handbuch Verfassungsrecht 24; WeRnER, KLECATSKY, Bundesverfassungsrecht 3f.; ADAMOVICH, FUNK, Österreichisches Verfassungsrecht 68.
}

namhafter Rechtshistoriker ${ }^{8}$ ist die junge Republik als zentralistischer Einheitsstaat begründet worden, in dem es zunächst weder Länder noch Landesgesetzgebung gab. Ermacora ${ }^{9}$ und Pernthaler $^{10}$ gehen demgegenüber von einer bündischen Gründung des neuen Staates aus, die auf zwei Ebenen und in zwei Etappen erfolgte. ${ }^{11}$ In Wien habe die Provisorische Nationalversammlung am 30. Oktober 1918 einen zentralistischen Einheitsstaat $\mathrm{zu}$ installieren unternommen. Gleichzeitig hätten sich aber in den Landeshauptstädten die Länder kraft ihrer wiedererlangten Verfassungshoheit als souveräne Staaten konstituiert. Erst ein Bund zwischen der Zentrale und den Ländern habe diese beiden Ebenen miteinander verschränkt und dabei die Souveränitätsfrage in der Schwebe gelassen: Die Länder hätten ihren Beitritt zur Republik Deutschösterreich erklärt, die Nationalversammlung habe diesen Beitritt angenommen. ${ }^{12}$

Die zentralistische Deutung beruft sich auf die $\S \S 1$ und 2 des Beschlusses der Provisorischen Nationalversammlung vom 30. Oktober 1918, StGBl. 1, über die grundlegenden Einrichtungen der Staatsgewalt. Nach ihm sei die oberste Gewalt des Staates Deutschösterreich durch die Provisorische Nationalversammlung ausgeübt worden, und zwar hinsichtlich der gesetzge-

\footnotetext{
${ }^{8}$ Vgl. Hellbling, Verfassungs- und Verwaltungsrechtsgeschichte 409; LEHNER, Verfassungs- und Verwaltungsgeschichte 259, 269; STAUDINGER, Ereignisse in den Ländern 63; VOLKER, Ausgleichsbestrebungen 23 (mit der Relativierung: „aus Wiener Sicht"). Ebenso der Sache nach KLEINWAECHTER, Von Schönbrunn nach St. Germain 84ff.; WALTER, Verfassung- und Verwaltungsrechtsgeschichte 262f. Weitere Nachweise bei KÖFNER, Revolutionen 132f.; schon in seiner Pionierarbeit heißt es: „Die Reihe könnte beliebig fortgesetzt werden“ (ebd. 160 Anm. 10).

${ }^{9}$ ERMACORA, Österreichischer Föderalismus 43ff.

10 PERNTHALER, Staatsgründungsakte 23ff.

11 Ähnlich BAUER, Österreichische Revolution 105ff.; ALTENSTETTER, Föderalismus in Österreich 11f.

12 Zusammenfassend PERNTHALER, Österreichisches Bundesstaatsrecht 285f.
} 
benden Gewalt von der Nationalversammlung selbst. Solange dieser Beschluss als gültige Verfassung vorausgesetzt werde, sei für eine konkurrierende Gesetzgebung durch die Landtage oder der an ihre Stellung tretenden Vertretungskörper kein Platz geblieben. ${ }^{13}$ Auch $\S 1$ des Gesetzes betreffend die Übernahme der Staatsgewalt in den Ländern ${ }^{14}$ habe an diesem Zustand nichts geändert, ordne es doch bloß an, dass an die Stelle der bisherigen Landtage die provisorischen Landesversammlungen treten..$^{15}$ Kompetenzen, insbesondere solche zur Abänderung der Landesordnungen, weise es diesen Landesversammlungen nicht $\mathrm{zu}^{16}$ - mit der Konsequenz, dass durch die Überleitungsvorschrift des $\S 16$ des Beschlusses vom 30. Oktober 1918 nur die Verwaltungsaufgaben rezipiert worden sein konnten. ${ }^{17}$

${ }^{13}$ KELSEN, Verfassungsgesetze 1, 18, 103; DERS., Verfassungsgesetze 3, 231; MERKL, Organisation 11 (Gesetzgebungsmonopol).

14 StGBl. 1918/24.

${ }^{15}$ Die bisherigen Landtage waren durch Art. 8 StGBl. 1918/5 abgeschafft worden. Hieraus ist aber für ein Gesetzgebungsmonopol der Provisorischen Nationalversammlung (entgegen LEHNER, Verfassungs- und Verwaltungsgeschichte 269) ebenfalls nichts zu gewinnen, zeigt doch die Beifügung „bisherige“, die bei den übrigen durch diese Bestimmung abgeschafften Institutionen (Delegationen, Herrenhaus) unterlassen worden war, dass die Nachfolgeeinrichtungen der Landtage, mithin die Landesversammlungen, nicht betroffen sein sollten.

${ }^{16}$ KELSEN, Verfassungsgesetze Bd. 1, 106ff. Nach dem Vorwort (VI) stammen diese Passagen freilich aus der Feder MERKLs. Anders KELSEN, Verfassungsgesetze 3, 136f.: Rezeption der Zuständigkeitsabgrenzung zwischen Reich und Ländern in der Monarchie.

${ }^{17}$ Kelsen, Verfassungsgesetze 1, 103f.; MerKL, Organisation 11, mit dem Hinweis, dass erst die Verfassung vom 14.3. 1919 (konkret gemeint: Art. 12 des Gesetzes über die Volksvertretung, StGBl. 179) den Landesversammlungen eine Gesetzgebungsbefugnis eingeräumt habe; ebenso WIDDER, Gesetzgebung 363f. Auch in Art. 12 werden freilich den Ländern keine Angelegenheiten in der Gesetzgebung zugewiesen, es wird vielmehr auf „alle Gegenstände, die nach der
Diese Deutung ist nur auf den ersten Blick plausibel. Nach $\S 1$ des Beschlusses über die grundlegenden Einrichtungen der Staatsgewalt ist der Provisorischen Nationalversammlung die oberste Gewalt des Staates vorbehalten. Das schließt selbständige Länder mit eigenem Gesetzgebungsrecht schon deshalb nicht aus, weil diese von der herrschenden Auffassung als autonome Körperschaften zur Selbstverwaltung gerechnet und als nichtstaatliche Entitäten dem Staat gegenüber gestellt wurden. ${ }^{18}$ Ebenso wenig ist die Anordnung der Ausübung der gesetzgebenden Gewalt durch die Nationalversammlung "selbst" in $\S 2$ gegen die Länder gerichtet. ${ }^{19}$ Wie sich aus dem Zusammenhang mit $\S 3$ ergibt, in dem die Regierungs- und Vollzugsgewalt einem Vollzugsausschuss namens Staatsrat übertragen wird, stellt sie lediglich klar, dass die Gesetzgebungskompetenz der Nationalversammlung delegationsfest konzipiert war. ${ }^{20}$ Folglich sprechen die besseren Argumente dafür, dass durch $\S 16$ des Beschlusses auch die Landesordnungen und mit ihnen eine Gesetzgebungsbefugnis der Länder in den neuen Staat Deutschösterreich übergeleitet worden sind, weil es insoweit keinen Widerspruch gab. ${ }^{21} \mathrm{Zu}$ dieser Auffassung

bestehenden Verfassung der Landesgesetzgebung unterliegen", verwiesen.

${ }^{18}$ Dazu sowie zum Stellenwert konkurrierender bundesstaatlicher Deutungsmuster vgl. SIMON, Föderalismus 276ff.; BRAUNEDER, Österreichische Verfassungsgeschichte $175 \mathrm{ff}$.

${ }^{19}$ Sie hatte wohl noch nicht einmal eine Spitze gegen den Kaiser. Anders SCHMITZ, Vorentwürfe 12; BERCHTOLD, Verfassungsgeschichte 1, 57.

${ }^{20}$ BRAUNEDER, Deutsch-Österreich 89.

${ }^{21}$ BRAUNEDER, Österreichische Verfassungsgeschichte; KÖFNER, Revolutionen 145f. und WERNDL, Kompetenzverteilung 18. Zutreffend ist indes, dass im Hinblick auf Art. 3 StGBl. 1918/5, wonach der Staatsrat alle „nach der Verfassung der im Reichsrate vertretenen Königreiche und Länder dem Kaiser" zugestandenen Rechte ausüben sollte, für Landesgesetze die Sanktion des Staatsrats erforderlich gewesen wäre: vgl. KELSEN, Verfassungsgesetze 3, 232; DERS., Stellung der Länder 102. Davon ging offenkundig auch die 
hat sich denn auch Kelsen in seiner letzten Einlassung in die Thematik durchgerungen. ${ }^{22}$

Wenig besser bestellt ist es um die föderalistische Sichtweise, nach der sich die Länder als selbständige Staaten mit unabgeleiteter Staatsgewalt konstituiert haben sollen. In den Beitrittserklärungen, die als Beleg für diese These herangezogen werden, ist von souveräner Staatlichkeit nichts zu lesen:23 Jene Länder, die ihre Zugehörigkeit zum neu entstandenen staatlichen Gebilde deklarierten, taten dies entweder als Provinzen ${ }^{24}$ oder als Länder, ${ }^{25}$ sie erkannten die Nationalversammlung vielfach ausdrücklich als übergeordnete Institution an, ${ }^{26}$ und sie nahmen bei alledem nicht selten auf den Beschluss

provisorische Landesordnung der Steiermark vom 6. 12.1918 (abgedruckt bei KELSEN, Verfassungsgesetze $3,186 f f$.$) aus, wenn nach ihrem \S 12$,die provisorische Landesversammlung bei der Ausübung der gesetzgebenden Gewalt innerhalb des ihr auf Grund der geltenden Gesetze zustehenden Wirkungskreises mitzuwirken" hatte.

${ }^{22}$ KELSEN, Entwicklung des Staatsrechts 148f., freilich ohne explizite Aufgabe älterer Auffassungen. $\mathrm{Zu}$ früheren Ansätzen einer Selbstkorrektur vgl. KÖFNER, Revolutionen 146f.

${ }^{23}$ Die Beitrittserklärungen sind gemeinsam mit den provisorischen Landesverfassungen abgedruckt bei KELSEN, Verfassungsgesetze 3, 181ff. Von Niederösterreich und Tirol ist keine Erklärung überliefert; mitunter wird aber die Entschließung des Tiroler Nationalrats vom 25.11. 1918 (abdruckt bei PERNTHALER, Staatsgründungsakte 70) als Beitrittserklärung qualifiziert.

${ }^{24}$ So Steiermark, Kärnten und Salzburg (gesonderte, eigenberechtigte Provinz), Deutschböhmen (eigenberechtigte Provinz) und Sudetenland (Provinz).

${ }^{25}$ So Oberösterreich (Land, das den anderen Ländern mit gleichen Rechten und Pflichten als Glied des Staates Deutschösterreich zur Seite tritt) und Vorarlberg (eigenes, selbständiges Land im Rahmen des Deutschösterreichischen Staates).

${ }^{26}$ In der Steiermark ,als derzeitige oberste staatliche Gewalt", in Kärnten „unter Wahrung des vollen Selbstbestimmungsrechts der Landesversammlung“, in Deutschböhmen als „ihre einzige und höchste gesetzgebende Körperschaft", in Oberösterreich "als derzeitige oberste staatliche Gewalt". der Provisorischen Nationalversammlung sowie einen Auftrag ihres Vollzugsausschusses Bezug. ${ }^{27}$ Die gleiche Sprache sprechen die provisorischen Verfassungen dieser Epoche, ${ }^{28}$ die bald eine gesonderte eigenberechtigte Provinz, ${ }^{29}$ bald ein selbständiges Gebiet mit eigener Gesetzgebung und Verwaltung ${ }^{30}$ konstituierten und die von einer Mitgliedschaft im deutschösterreichischen Staat ausgingen. ${ }^{31}$ Mit dieser Zurückhaltung in der eigenen Bezeichnung korrespondierte eine Selbstbeschränkung im Wirkungskreis: Weder die Beitrittserklärungen noch die provisorischen Verfassungen sprachen den Ländern mehr als jene Rechte zu, die ihnen nach den Landesordnungen der Monarchie zukamen. ${ }^{32}$ Vorarlberg und Tirol bildeten keine Aus-

${ }^{27}$ So die Steiermark, Kärnten und Salzburg.
${ }^{28}$ Abgedruckt bei KELSEN, Verfassungsgesetze 3,
$181 \mathrm{ff}$.
${ }^{29}$ Salzburg (Kapitel I, Verf. $\S 1$ ).
${ }^{30}$ Vgl. Deutschböhmen $(\S 1)$.
${ }^{31}$ Aufgrund der zeitgleich bzw. uno actu beschlossenen Beitrittserklärungen kommt sie in den Verfassungen regelmäßig nur indirekt zum Ausdruck. Vgl. für die Steiermark $\S 7$ (Angelobung des Landeshauptmannes durch den Präsidenten des Staatsrates auf die deutschösterreichische Republik), für Kärnten III. Hauptstück Z 2 (Erwartung der Wahrung des Rechts Kärntens auf zollfreien Verkehr insbesondere mit Triest durch den deutschösterreichischen Staat), für Deutschböhmen $\S 1$ (Gebiet des Staates Deutschösterreich), für das Sudentenland $\S 10$ (Bindung an die Gesetze, Verordnungen, Verfügungen und Erkenntnisse der Nationalversammlung und ihrer Vollzugsorgane).

${ }^{32}$ Vgl. für Kärnten II. Hauptstück Z 2 der Beitrittserklärung (Rechte und Pflichten nach den bisher geltenden Gesetzen); für die Steiermark 2. Kap. Z 1 Beitrittserklärung und $\S 1 \mathrm{LV}$; für Salzburg Kap. II der Beitrittserklärung (Rechtsnachfolge des Landes in bisher geltenden Rechte und Pflichten, Überleitung der bisher geltenden Verfassungsgesetze); für Deutschböhmen Art. 1 Beitrittserklärung (Rechte und Pflichten, die in der böhmischen Landesordnung niedergelegt sind); für Sudetenland $§ 3$ (die Landesversammlung tritt an die Stelle der Landtage nach deren sich durch die Landesordnung ergebenden Bestande). 
nahme. Wenn sich in Vorarlberg die Landesversammlung zur gesetzgebenden Körperschaft ausrief und die Selbständigkeit des Landes erklärte, so war damit eine Stellung ,[w]ie in anderen Kronländern" und die Abtrennung von Tirol gemeint, keine Souveränität. Desgleichen wurde in der Entschließung des Tiroler Nationalrates vom 25. November 1918 über das Verhältnis zur Zentralregierung ${ }^{33}$ nicht auf Souveränität, sondern auf „Autonomie des Landes“ gepocht, und es wurde auf "die endgiltige Regelung der staatsrechtlichen Gestaltung des Landes" durch die noch zu wählenden Volksvertretungen verwiesen.

Aber, so mag man einwenden, haben die Beitrittserklärungen und deren Annahme durch die provisorische Nationalversammlung ${ }^{34}$ nicht eine bündische Note? Nicht nur die Innsbrucker Schule, auch Kelsen hat hierin die "Tendenz einer bundesstaatlichen Struktur", die "Vorstellung einer vertragsmäßigen Konstituierung des Staates“ erblickt, ihr "symptomatische Bedeutung" zugemessen und einen "Geburtsfehler" des jungen Staates diagnostiziert, "dessen verhängnisvolle Wirkungen die Existenz dieses Gemeinwesens ständig bedrohen“. ${ }^{35}$

Was im Rückblick als bündisches Element erscheinen mag, hatte im zeitgenössischen Kontext einen gänzlich anderen Sinn. Deutschösterreich verfolgte das Ziel, alle Territorien mit deutscher Besiedelung unter seine Gebietshoheit zu brin-

\footnotetext{
33 Wiedergegeben bei PERnthaler, Staatsgründungsakte 70 .

${ }^{34}$ Beschluß der Provisorischen Nationalversammlung für Deutschösterreich vom 12.11. 1918 StGBl. 23, betreffend die feierliche Beitrittserklärung der Länder, Kreise und Gaue des Staatsgebietes, mit der die Erklärungen dieser namentlich nicht genannten Gebiete zur Kenntnis genommen und "diese Gebiete des Staates unter den Schutz der ganzen Nation“ gestellt wurden.

${ }^{35}$ KELSEN, Verfassungsgesetze 1, 72 (erstes bis drittes Zitat); DERS., Österreichisches Staatsrecht 102 (viertes und fünftes Zitat).
}

gen, ${ }^{36}$ und hatte daher angesichts der WilsonDoktrin außenpolitisch allen Grund, auf das Selbstbestimmungsrecht der Nationen zu setzen. Die Beitrittserklärungen dienten exakt diesem Zweck: ${ }^{37}$ Förmliche Willensbekundungen der beanspruchten deutschsprachigen Gebiete sollten die Ansprüche der anderen Nachfolgestaaten der Monarchie abwehren und die Alliierten bei ihren eigenen Prinzipien nehmen helfen. ${ }^{38}$ Wohl deshalb ist die förmliche Festlegung des Staatsgebietes erst nach Einlangen der Beitrittserklärungen insbesondere der Provinzen auf tschechischem Gebiet erfolgt. ${ }^{39}$

Im Übrigen geht die Vorstellung antagonistischer Revolutionen in Wien und in den Ländern, die auf miteinander unvereinbare Modelle von Staatlichkeit gerichtet gewesen sein sollen, an den historischen Fakten vorbei. ${ }^{40}$ Diese vermit-

\footnotetext{
${ }^{36} \mathrm{Vgl}$. die Vorlage des Staatsrates betreffend den Entwurf einer Staatserklärung über Umfang, Grenzen und Beziehungen des Staatsgebietes (3 BlgProvNV) der ersten Vorlage überhaupt -, den Antrag des Verfassungsausschusses betreffend diese Staatserklärung (21 BlgProvNV) sowie den Antrag der Abgeordneten Marckl und Genossen betreffend die Sicherung des Selbstbestimmungsrechtes und Schutzes deutscher Siedlungen vom 30. 10. 1918 (31 BlgProvNV).

${ }^{37} \mathrm{Vgl}$. ABLeitinger, Grundlegung 153; BRAUnEDER, Gesamtstaat - Länder 34; SCHMITZ, Vorentwürfe 21f.

${ }^{38}$ In der Erklärung der Landesversammlung des Sudetenlandes vom 16.11. 1918 wird denn auch einerseits das Selbstbestimmungsrecht der slawischen Völker vorbehaltslos anerkannt, andrerseits aber die „Wahrung unseres unverkürzbaren Selbstbestimmungsrechtes" eingefordert (KELSEN, Verfassungsgesetze 3, 224).

${ }^{39}$ Vgl. das Gesetz vom 22. 11. 1918, StGBl. 40 über Umfang, Grenzen und Beziehungen des Staatsgebietes von Deutschösterreich und die „im Namen des deutschösterreichischen Volkes zur Sicherung seiner Wohnstätten wie seiner Stellung unter den anderen Staaten und Völkern“ beschlossene korrespondierende „Staatserklärung“, StGBl. 1918/41. Ebenfalls einen Bezug zu den Beitrittserklärungen herstellend ERMACORA, Österreichischer Föderalismus 43.

${ }^{40}$ Eingehend KÖFNER, Revolutionen 134ff.; seiner Einschätzung folgend BRAUNEDER, Gesamtstaat Länder 35f.; SCHMITZ, Vorentwürfe 16; HAUTMANN,
} 
teln vielmehr den Eindruck eines harmonisches Konzerts, veranstaltet von den politischen Parteien, die an einem Strang zogen und im Staat wie in den Ländern synchron vorgingen, orchestriert von der Staatskanzlei und dirigiert durch deren Leiter: Karl Renner hatte die Beitrittserklärungen organisiert und den Ländern Muster übermittelt, ${ }^{41}$ nachdem zuvor die Vorgangsweise mit Vertretern der Länder abgesprochen worden war. ${ }^{42}$ Weder agierte die Zentrale gegen die Länder, noch die Länder gegen die Zentrale. ${ }^{43}$

Entstehungsbedingungen 35, und SCHEFBECK, Entstehung des Bundesrates 313. Vorbehalte hingegen bei ÖHLINGER, Rezension 114f.

${ }^{41}$ ERMACORA, Materialien 1, 39. Das erklärt, weswegen sich die Anfang November abgegebenen Erklärungen im Aufbau und im Inhalt gleichen: KÖFNER, Revolutionen 140.

42 BERCHTOLD, Verfassungsgeschichte der Republik Österreich 39ff.; BRAUNEDER, Deutsch-Österreich 64ff.; KÖFNER, Revolutionen 137f. Vgl. auch die (gewiss subjektiv gefärbte und hinsichtlich der Daten nicht korrekte) Rede Renners im Nationalrat am 22. 10. 1929, StProtNR III. GP 2877f.: „Es war Mitte November des Jahres 1918, da erschienen vor mir im Geleit des Staatesrates Dr. Stephan Licht - den ich mir, offenbar um eine bolschewikische Diktatur einzurichten, als notorischen Vertrauensmann des großen Bürgertums für alle Gesetzgebungsfragen attachiert hatte - die Herren Dr. Stölzel von Salzburg, Oberkurator Steiner, der damalige Landmarschall von Niederösterreich, und noch andere Herren, welche in den damaligen Landesverwaltungen tätig waren, und fragten: Was soll mit uns Ländern, was mit den Statthaltereien geschehen?"

${ }^{43}$ Ebenso KÖFNER, Revolutionen 151, BERCHTOLD, Verfassungsgeschichte der Republik Österreich 45f., der von Gleichartigkeit und Parallelität der Vorgangsweise spricht, und BRAUNEDER, DeutschÖsterreich 67: „Das Vorgehen in den Ländern war damit nicht nur unter diesen, sondern auch mit der Zentralgewalt akkordiert." Anders nuançiert ÖHLINGER, Entstehung des Bundesstaates 42ff., wenn er auf Berufungen auf das Selbstbestimmungsrecht sowie Ineffektivität des Weisungsrechts der Zentralregierung verweist. Letzteres war jedoch gar nicht umfassend konzipiert (vgl. KÖFNER, Revolutionen 147f.), die ersteren schließen an die Wilson-Doktrin an
Bis Jahresende sollte sich an diesem Staat wie Länder umspannenden Konsens über die Übernahme der Selbstverwaltungsstrukturen bei gleichzeitiger Stärkung der Länder durch Beseitigung des „Doppelgeleises“ der Verwaltung in den Ländern nichts ändern. Zwar führten die Interessengegensätze in der Ernährungsfrage und das Insistieren der Nationalversammlung auf der Sanktionierung der Landesgesetze ${ }^{44} \mathrm{zu}$ ersten Konflikten zwischen Wien und den Landeshauptstädten. ${ }^{45}$ Bereits die oberösterreichische Beitrittserklärung bringt dieses abgekühlte Verhältnis zum Ausdruck. ${ }^{46}$ Zeitgleich ist in der Tagespresse eine erste leise Stimme zu registrieren, die als Alternative zum gegenwärtigen $\mathrm{Zu}$ stand eine Föderation ins Spiel brachte. ${ }^{47}$ Da sie

und stellen zwar Akte autonomer Gebietskörperschaften, aber keine Staatlichkeitsproklamationen dar. ${ }^{44}$ Zum verfassungsrechtlichen Hintergrund vgl. oben Anm. 21. Der Unmut der Länder hierüber ist plastisch artikuliert in der Titulierung Renners als „imperator redivivus" durch Ender auf der 3. Länderkonferenz: vgl. ERMACORA, Materialien 1, 22.

${ }^{45}$ KÖFNER, Revolutionen 152ff.; STAUDINGER, Ereignisse in Ländern 72f.; ÖHLINGER, Entstehung des Bundesrates 43 .

${ }^{46} \mathrm{Zu}$ ihren Besonderheiten KÖFNER, Revolutionen $141 f$.

${ }^{47}$ So SEIPEL, Wesen des demokratischen Staates 57: „Aber definitiv wird der neue Staat Deutschösterreich nicht anders zustandekommen können, als durch Föderation der Kronländer, deren Bevölkerung es ja an Organen zur legalen Willensäußerung durchaus nicht fehlt." Der Kontext dieses unter der Seitenüberschrift „Nationalversammlung oder sozialistische Diktatur in Deutschland?" erschienenen Artikels zeigt jedoch, dass es sich um eine Nebenbemerkung handelt. Seipels Thema ist die Demokratie, sein Anlass die absehbaren Wahlen, sein Problem die politische Dominanz der Sozialdemokraten, seine Argumentationskette die folgende: Demokratie schließt nicht nur die Beherrschung des Volkes durch nicht vom Volk zur Herrschaft Berufene, es schließt auch „die Beherrschung einer Nation, einer Bevölkerungsgruppe, einer Klasse und in letzter Vollendung auch einer Partei durch eine andere aus“ (54). „Der einzige demokratische Weg der Staatenbildung ist der der Föderation“ (55). „Doch wir lesen in den neuen Grundgesetzen nichts von dem" (56). In der endgültigen 
ohne Echo blieb, kann man von einer Bundesstaatsdiskussion aber noch nicht sprechen.48 Soweit bis Jahresende 1918 in den Parteien Überlegungen über die Ausgestaltung der endgültigen Verfassung angestellt wurden, war weiterhin Autonomie die dominierende Losung. ${ }^{49}$ Unverdächtiges Zeugnis für diese These gibt das Wahlprogramm der Christlichsozialen vom 15. Dezember $1918 \mathrm{ab}$, das sich der Autonomie der Länder wie der Gemeinden verschreibt und ihren Ausbau fordert. ${ }^{50}$

Verfassung soll und muss hingegen jedes Kronland, vor allem aber jeder Stand „volle Gewähr" erhalten, frei zu sein, mitbestimmen zu können und nicht zum Knecht der anderen zu werden (57f.). Auf die Föderation aber nimmt der an Bundesstaatlichkeit notorisch nur instrumentell interessierte Autor nicht zuletzt deshalb Bezug, weil es ihm Gelegenheit gibt, frühere Forderungen Renners in diese Richtung im Wortlaut wiederzugeben (56).

${ }^{48}$ Eine solche gab es aus Anlass der Deklaration Kaiser Karls, die Monarchie Cisleithaniens in einen Bundesstaat umwandeln zu wollen (vgl. Wiener Zeitung Nr. 240 vom 17. 10. 1918 und das nachfolgende breite Medienecho). Aus Anlass der Aufrichtung der deutschösterreichischen Republik gab es sie nicht, wie überhaupt die Föderalisierungsdebatten der Monarchie (vgl. etwa die auf eine Rundfrage der Österreichischen Zeitschrift für öffentliches Recht erstatteten, im 3. Jahrgang 1916 abgedruckten Gutachten) auf die Diskussion nach 1918 keinen Einfluss hatten. Das ist umso bemerkenswerter, als die Bundesstaatsthematik in der Öffentlichkeit noch präsent gewesen sein musste. Der Grund liegt wohl darin, dass es in der Republik kein Nationalitätenproblem mehr zu lösen gab.

${ }^{49}$ Pointierend KÖFNER, Revolutionen 156: „Die wirtschaftliche Not, die Erfahrungen des Krieges und der drohende Hunger ließen in allen Ländern einen starken Landesegoismus entstehen, der von theoretisierenden Verfassungshistorikern dann als Landespatriotismus und Souveränitätsbewußtsein interpretiert wurde!"

${ }^{50}$ Reichspost vom 25. 12. 1918, 1 = BERCHTOLD, Parteiprogramme 356: „Die christlichsoziale Partei anerkennt das Recht der Länder und Gemeinden auf ihre Autonomie und fordert deren Ausbau.“

\section{Februar 1919 bis September 1919: Positionierung der Länder}

Nach dem Jahreswechsel änderte sich das Bild: Nunmehr erwachten die Länder und übernahmen die Initiative. ${ }^{51}$ Das machte sich auf drei Ebenen bemerkbar.

Erstens sprachen ihre provisorischen Landesverfassungen teilweise eine andere, nunmehr eindeutig bundesstaatliche Sprache. ${ }^{52}$ Die Vorarlberger Verfassung vom 14. März $1919^{53}$ proklamierte die Selbständigkeit des Landes und erkannte als Schranke dieser Selbständigkeit in $\S 1$ nur "die Verfassung des Bundesstaates" an offen lassend, ob dies denn ein schweizerischer, ein deutscher oder ein österreichischer Bundesstaat sei. ${ }^{54}$ Zudem erfolgte die Anerkennung einer das Land bindenden Verfassung des Bundesstaates nach $\S 51$ unter der Bedingung, dass der Bundesstaat dem Land „sein Gebiet, seine Selbständigkeit innerhalb der Schranken des $\S 1$ dieser Verfassung, die Freiheit, die Rechte des Volkes und die verfassungsmäßigen Rechte der Volksgenossen, sowie die Rechte und Befugnisse, die das Volk seinen Vertretern übertragen hat, gewährleistet". Das ist ein alemannischer, deutlich durch die schweizerische Bundesverfassung 1874 inspirierter Zungenschlag.

Vorarlberg stand aber mitnichten allein, auch andere Länder traten selbstbewusster auf. Nach Art. I des Gesetzes über die Grundzüge der Landesvertretung vom 18. März 191955 übte das Land Oberösterreich als selbständiges Land alle Rechte aus, welche nicht durch ausdrückliche Vereinbarung der Gewalt eines Bundesstaates übertragen worden waren. Art. II des Kärntner Gesetzes über die Einberufung des verfassung-

\footnotetext{
51 Altenstetter, Föderalismus in Österreich 14.

52 Ebenso SchMiTZ, Vorentwürfe 39f.

${ }^{53}$ LGB1. 1919/22.

${ }^{54}$ Vgl. BRAUNEDER, Deutsch-Österreich 229.

${ }^{55}$ LGVBl. 1919/23.
} 
gebenden Landtages ${ }^{56}$ sprach dem Kärntner Landtag die Befugnis zu, „über die Frage des Zusammenschlusses der deutschösterreichischen Länder zu einem Gesamtstaate oder des Anschlusses des Landes an einen anderen Staat durch Volksabstimmung entscheiden zu lassen“. Zweitens traten die westlichen Länder mit Vorarlberg an der Spitze auf der 3. Länderkonferenz, die am 31. Jänner und am 1. Februar 1919 in Wien stattfand, mit der Forderung nach Bundesstaatlichkeit auf. Renners die Konferenz einleitendes Plädoyer für Selbstregierung nach englischem Muster und für eine Weiterentwicklung der Autonomie der Länder unter Beseitigung der Doppelgleisigkeiten im Verwaltungsapparat $^{57}$ traf auf wenig Zustimmung. Ender verwies auf die Schweiz als besseres wie näheres Vorbild und forderte eine klassische, schiedliche und friedliche Scheidung der Kompetenzen sowie die Garantie, dass die Länder ihre Zuständigkeiten frei ausüben dürfen, unbehelligt von Rahmengesetzen der Zentrale und vergleichbaren Gängelungen. ${ }^{58}$ Die übrigen Landeshauptleute und die meisten sonstigen Debattenredner pflichteten ihm in der Einschätzung bei, dass sich die staatliche Einheit nur auf Grundlage einer föderativen Verfassung aufrechterhalten lasse. ${ }^{59}$ In seiner Replik gestand denn auch Renner zu, dass die Schweiz für die Verfassung ein gutes Vorbild abgebe. ${ }^{60}$

\footnotetext{
${ }^{56}$ LGBl 1919/20.

${ }^{57}$ ERMACORA, Materialien 1, 12ff. Anders noch seine Position unter der Monarchie: vgl. das Plädoyer für Bundesstaatlichkeit bei RENNER, Selbstbestimmungsrecht 1, 232ff. Vgl. zu Renners Vorstellungen auch BRAUNEDER, Karl Renners Entwurf 78ff.; SCHMITZ, Briefe 55ff., 133ff.

${ }^{58}$ ERMACORA, Materialien 1, $22 \mathrm{ff}$.

${ }^{59} \mathrm{Vgl}$. die Wortmeldungen von Hauser, Mittelberger, Pusch, Angerer, von Steiner, Frank und Rehrl (ERMACORA, Materialien 1, 25ff.). Nur die sozialdemokratische Seite zeigte für die Pläne und Wünsche Renners Verständnis.

${ }^{60}$ ERMACORA, Materialien 1, 38. Schon in seiner Einleitung hatte Renner konzediert, dass bei Scheitern des
}

Drittens setzten sich die Länder im Frühjahr 1919 mit ihrer Forderung nach einem Bundesstaat innerhalb der Christlichsozialen durch. ${ }^{61}$ Im Aktionsprogramm vom 6. März 1919 war das Wort Bundesstaat noch vermieden worden. Es sah für die neue Verfassung zwar eine feste Kompetenzverteilung zwischen Staat und Ländern mit einer Generalklausel zugunsten der Länder vor und forderte demokratische Länderverfassungen, die vom Staate zu gewährleisten waren. ${ }^{62}$ Eine Länderkammer sucht man indes vergeblich. Eine solche war zwar ursprünglich postuliert, ${ }^{63}$ in der Vollversammlung des Klubs am 3. März 1919 aber noch abgelehnt worden. Der gut zwei Monate später eingebrachte erste Verfassungsentwurf der Christlichsozialen sah hingegen einen Bundesstaat mit weitreichenden Befugnissen der Länder und einer starken Vertretung der Länder im Bunde vor. ${ }^{64}$

Anschlusses an Deutschland die bundesstaatliche Option nach Schweizer Muster den Vorzug verdiene (ebd. 17).

${ }^{61} \mathrm{Vgl}$. SEIPEL, Wesen des demokratischen Staates 74ff.

${ }^{62}$ Vgl. Reichspost vom 6.3. 1919, 4 = BERCHTOLD, Parteiprogramme 360f.

${ }^{63}$ Vgl. den in der Reichspost vom 28. 2. 1919, 1, abgedruckten Entwurf. Die gestrichene Passage lautete: „Um die Rechte der Länder zu sichern, soll ein von ihnen zu beschickendes Länderhaus mit genau umschriebenen Befugnissen der Nationalversammlung an die Seite gestellt werden."

${ }^{64}$ Vgl. den am 14. 5. 1919 eingebrachten Entwurf 231 BlgKonstNV, abgedruckt bei ERMACORA, Quellen $29 \mathrm{ff}$. Eckpunkte dieses Entwurfs waren der freie Zusammenschluss der Länder zu einem Bundesstaat (Art. 3), die Selbständigkeit der Länder, soweit sie nicht durch die Bundesverfassung eingeschränkt war, sowie die Ausübung aller Rechte durch sie, soweit die Bundesverfassung sie nicht der Bundesgewalt übertragen hatte (Art.4), die taxative Aufzählung der Bundeskompetenzen in Anlehnung an das StGG über die Reichsvertretung (Art. 14) sowie eine Generalklausel zu Gunsten der Länder (Art. 15), die; Aufzählung jener Landesangelegenheiten, in denen die Bundesregierung mitwirken darf (Art. 16, 54), ein Ständehaus als Länder- und Rätekammer mit drei Vertretern pro Land sowie einer eine offen gelassenen Anzahl von Vertretern der Räteorganisationen (Art. 20), das Er- 
Die Kehrtwendung der Länder, die zuvor mit einem Autonomiekonzept einverstanden gewesen waren und nun prononçiert föderalistische, mitunter sogar offen separatistische Pläne verfolgten, geht zum einen auf die Mitte November 1918 beginnende Entfremdung zurück. Zum anderen hat sie auch in der Hoffnung Tirols, als unabhängiger neutraler Staat seine Landeseinheit bewahren zu können, ${ }^{65}$ und in der Vorarlberger Anschlussbewegung 66 ihre Gründe.

Die Sozialdemokraten warteten in dieser Phase ab. Nach ihrem Wahlsieg im Februar 1919 sowie infolge der Proklamation der ungarischen Räterepublik im April befanden sie sich in einer Situation der Stärke, die sie in der Arbeits- und Sozialgesetzgebung umzusetzen bestrebt waren, ${ }^{67}$ Die Verfassungsfrage stand nicht im Zentrum ihres Interesses, und ihre Position zur Bundesstaatlichkeit war ambivalent. Das Aktionsprogramm vom 26. Februar 191968 hatte sich gegen den Widerstand Renners dem Einkammersystem verschrieben. Mit dem Satz „Reichsrecht bricht Landesrecht" verlangte es aber auch die Einführung eines bundesstaatlichen Instituts, und die Forderungen nach einer Kompetenz des Staates für die wirtschaftliche und soziale Gesetzgebung sowie für den Ernährungsdienst machten deutlich, dass an eine Abschaffung der Landesgesetzgebung nicht gedacht war. Selbst der programmatische, als Reaktion auf den Verfassungsentwurf der Christlich-

fordernis übereinstimmender Beschlüsse beider Häuser bzw. eine Zweidrittelmehrheit in gemeinsamer Sitzung für Bundesgesetze (Art. 27), schließlich die Entscheidung von Streitigkeiten zwischen Bund und Ländern sowie zwischen den Ländern untereinander durch den Verfassungsgerichtshof (Art. 55 Abs. 2).

${ }^{65}$ HANISCH, Schatten des Staates 269; SCHEFBECK, Entstehung des Bundesrates 314.

${ }^{66} \mathrm{Zu}$ ihr BERCHTOLD, Verfassungsgeschichte der Republik Österreich 106ff.

${ }^{67}$ BAUER, Österreichische Revolution 129 f; HAUTMANN, Entstehungsbedingungen 38f.

${ }^{68}$ Volkstribüne vom 26. 2. 1919 = BERCHTOLD, Parteiprogramme 233f. sozialen entstandene Artikel von Austerlitz bringt in meinen Augen keine unbedingte Ablehnung von Bundesstaatlichkeit zum Ausdruck. ${ }^{69}$ In ihm ist betont, dass die Länder niemals über mehr Autonomie verfügt hätten als unter der Republik und dass die Rede von einem zentralistischen System entweder auf Unkenntnis oder Böswilligkeit basiere. ${ }^{70}$ Austerlitz schränkte ein, dass „die natürliche, aus den Bedürfnissen unseres Lebens abgeleitete Ordnung" der einheitliche Staat wäre, der sich in Kreise teilt, die in ihrer Selbstverwaltung in die Tiefe gingen und nicht wie die überlieferten Länderverwaltungen an der Oberfläche blieben. Schon im Folgesatz stellte er jedoch klar, dass er diese Forderung angesichts der Geschichte für unrealisierbar hielt. ${ }^{71}$

Die Staatskanzlei legte sich ebenfalls schon in dieser Phase auf einen Bundesstaat fest und richtete sich im Übrigen auf alle Eventualitäten ein. Renner besprach sich vor seiner am 12. Mai 1919 erfolgten Abreise nach St. Germain mit Kelsen über die Grundlinien der künftigen Verfassung, die bundesstaatlichen Charakter haben und eine parlamentarische Republik einrichten sollte. ${ }^{72}$ Auftragsgemäß arbeitete Kelsen über den Sommer 1919 mehrere Entwürfe aus, die teilweise ausgesprochen länderfreundliche Züge tragen. ${ }^{73}$ Das gilt vor allem für seinen ersten

\footnotetext{
${ }^{69}$ Anders MATZKA, Sozialdemokratie und Verfassung 62, der in Austerlitz den einzigen konsequent gegen föderalistische Initiativen auftretenden Sozialdemokraten sieht.

${ }^{70}$ AusTERLITZ, Staat und Länder 330f.

${ }^{71}$ Der Artikel klingt denn auch mit der Prognose aus, dass die Entwicklung der ehedem von Großgrundbesitzern beherrschten Landesversammlungen $\mathrm{zu}$ grunddemokratischen und dadurch selbstbewussten Organisationen das richtige Gleichgewicht zwischen Ländern und Staat schon herbeiführen würde, würde nicht der Antagonismus zwischen Hauptstadt und Land als Störfaktor wirken.

${ }^{72}$ Vgl. SCHMITZ, Vorentwürfe 44 m.w.N.

${ }^{73} \mathrm{Vgl}$. schon ERMACORA, WIRTH, Bundesverfassung und Kelsen 40ff.; ABLEITINGER, Grundlegung 167f.
} 
Entwurf. ${ }^{74}$ In ihm ist etwa von „souveränen Ländern" die Rede, wozu sich in weiterer Folge nicht einmal der Tiroler Verfassungsentwurf versteigen sollte, ${ }^{75}$ Einflussnahmemöglichkeiten des Bundes auf dem Weg der Landesgesetzgebung - im Entwurf der Christlichsozialen und im Tiroler Entwurf etwa durchaus existent ${ }^{76}$ fehlen ebenso wie die Anordnung eines Vorrangs des Bundesrechts vor dem Landesrecht, ${ }^{77}$ und die Kompetenzausstattung des Bundes ist vergleichsweise schmal. ${ }^{78}$

\section{Oktober 1919 bis März 1920: Dialog zwischen der Zentral- bürokratie und den Ländern}

Das Zuwarten hatte im Herbst 1919 ein Ende. Mit dem Staatsvertrag von St. Germain waren die Entscheidungen gegen den Anschluss Österreichs an Deutschland und gegen die geplante Einbeziehung Deutschböhmens und des Sudetenlandes gefallen, die zunächst zurückgestellte Verfassungsfrage stand wieder auf der Tagesordnung. Damit brach eine weitere Phase an, die von Oktober 1919 bis März 1920 andauerte und in der die Zentralbürokratie als Akteur und Dialogpartner der Länder ins Spiel kam. An ihrem Beginn standen Vorentscheidungen, die den Ländern, ohne und gegen die keine Verfassung zu machen war, Entgegenkommen und Gesprächsbereitschaft signalisieren sollten.

Erstens enthielt das zwischen der Christlichsozialen Vereinigung deutscher Abgeordneter und dem Verband der Sozialdemokratischen Abgeordneten abgeschlossene Koalitionspro-

\footnotetext{
74 SCHMITZ, Vorentwürfe 49.

${ }^{75}$ Vgl. Art. I, II, V Entwurf I, abgedruckt bei SCHMITZ, Vorentwürfe 114ff., und ERMACORA, Entstehung 4, $62 \mathrm{ff}$.

${ }^{76} \mathrm{Vgl}$. Art. 48 und 49 bzw. Art. XVI.

77 Anders Art. 10 Entwurf II, III und VI bzw. Art. 9 Entwurf V.

${ }^{78} \mathrm{Vgl}$. Art. Vf. Entwurf I und IV, Art. 8f. Entwurf II, III und VI, Art. 7f. Entwurf V.
}

gramm vom 17. Oktober 191979, eine Festlegung auf den Bundesstaat. In der neuen Verfassung sollten die Bundeskompetenzen, zu denen Auswärtiges, Justiz, Wirtschaft, Soziales, Hochschule, Aufteilung der Steuerquellen sowie Rahmensetzung für die Schule und den Agrarbereich gehören sollten, taxativ aufgezählt werden. Außerdem wurde ein Zweikammersystem nach deutschem Muster in Aussicht gestellt, wobei bei Verweigerung der Zustimmung des Bundesrats der Volksentscheid eingeholt werden sollte. Verheißungen rascher Demokratisierung und Vereinfachung der Verwaltung sowie der Schaffung einer instanzenmäßig gegliederten Verwaltungsrechtspflege nach preußischem Muster runden das Bild ab und verraten die Handschrift Renners. ${ }^{80}$ Diese Weichenstellungen bargen für die Länder keine Überraschungen. Renner hatte sie ihnen schon auf der am 12. und 13. Oktober 1919 stattfindenden 7. Länderkonferenz eröffnet, und die Länder hatten dort gleichwohl auf institutioneller Einbindung in die Ausarbeitung und Beschlussfassung der Verfassung beharrt. ${ }^{81}$

Zweitens enthielt die Kabinettsliste ein wichtiges, in dieser Form nicht erwartetes Signal. Mit der Leitung des neu eingerichteten Staatsamts für Verfassungs- und Verwaltungsreform wurde mit dem Tiroler Geschichtsprofessor Michael Mayr ein überzeugter Föderalist betraut, der in den Ländern Vertrauen genoss. Diese Rechnung ging mehr als nur ein Stück weit auf. Unter

\footnotetext{
${ }^{79}$ Reichspost vom 18. 10. 1919, 1.

${ }^{80} \mathrm{Zu}$ seiner Rolle allgemein und speziell in dieser Phase ABLEITINGER, Grundlegung 154ff., 168ff.

${ }^{81} \mathrm{Vgl}$. ERMACORA, Materialien 1, 62ff.; Replik von Renner, ebd. 70f.: „In Wahrheit wird die Verfassung beschlossen werden durch die überwältigende Mehrheit der Parteien hier und dort, so daß wir, glaube ich, der theoretischen Erörterung und juristischen Vorbehalte enthoben sind“. - Zur vorangegangenen Forderung des Tiroler Landtages und ihrer Aufnahme durch andere Landtage BERCHTOLD, Verfassungsgeschichte der Republik Österreich 200ff., 214ff.
} 
Mayr intensivierte die Staatskanzlei ihre Arbeiten an der Verfassung, insbesondere stimmte sie die Kompetenzverteilung mit den Staatsämtern ab. ${ }^{82}$ Tirol legte mit Unterstützung von Vorarlberg, Salzburg, Oberösterreich und Niederösterreich den von Falser ausgearbeiteten und im Landtag am 18. Dezember 1919 beschlossenen Verfassungsentwurf vor, um Wien in Zugzwang zu bringen. ${ }^{83}$ Innerhalb der Koalition verhinderten jedoch Auffassungsunterschiede zwischen den Parteien über Schlüsselkompetenzen sowie über den Bundesrat eine Einigung. Dass nicht völliger Stillstand herrschte, ist Mayr zu verdanken, der mit einer Besuchsdiplomatie begann, mit den Ländern Gespräche über die Verfassung führte und die weit auseinander klaffenden Ansichten der Christlichsozialen in Wien und in den Ländern auf eine mittlere Linie brachte. ${ }^{84}$

Mayr nahm in der Folge, wenngleich gegen den Widerstand einiger Kollegen im Kabinett und daher nur als "Privatmann", an der von den Ländern einberufenen Länderkonferenz in Salzburg vom 15. bis 17. Februar 1920 teil. Sein Erscheinen wurde von den Ländern dadurch honoriert, dass sein „Privatentwurf" und nicht der Tiroler Entwurf die Beratungsgrundlage abgab. Die Konferenz kulminierte in einer Umfrage unter den versammelten Ländervertretern, ob Österreich als Bundesstaat oder als Einheitsstaat eingerichtet werden sollte. ${ }^{85}$ Sie zeigte, dass die Christlichsozialen und die Großdeutschen einhellig eine föderative Verfassung favorisierten, dass aber auch die Sozialdemokraten in den Ländern ihr einiges abgewinnen konnten. In

\footnotetext{
82 Dokumentation bei ERMACORA, Entstehung 2, 59ff.

${ }^{83} \mathrm{Zu}$ Vorgeschichte und Bedeutung des FalserEntwurfs siehe ERMACORA, Quellen 66, sowie VOLKER, Ausgleichsbestrebungen 119ff.

${ }^{84} \mathrm{Zu}$ den Stationen seiner Gesprächsreisen VOLKER, Ausgleichsbestrebungen $122 \mathrm{ff}$; Übersicht über die Reaktionen der Länder bei ScHMITZ, Verfassungsgespräche 24ff.

${ }^{85}$ ERMACORA, Materialien 1, $160 \mathrm{f}$.
}

Wien, Niederösterreich und der Steiermark präferierten sie den Bundesstaat unter der Bedingung voller Demokratie und wirtschaftlicher Entwicklung, in Oberösterreich sahen sie hierin eine gangbare Alternative, und nur in den westlichen Ländern, in denen ihr politischer Einfluss marginal war, brachen sie dem Einheitsstaat mit neuen wirtschaftlichen Verwaltungsgebieten die Lanze. Im Übrigen stand die Länderkammer im Zentrum der Debatten: Die kleinen und westlichen Bundesländer forderten die paritätische Vertretung der Länder, die großen und östlichen eine Vertretung der Größe nach, und einige Vertreter der Sozialdemokraten lehnten eine zweite Kammer weiterhin ab. Die Systematik der Kompetenzverteilung wurde hingegen zwischen Christlichsozialen und Deutschfreiheitlichen grundsätzlich konsentiert, lediglich die Sozialdemokraten wollten sich in dieser Frage noch nicht festlegen. 86

\section{April 1920 bis Juli 1920: Dominanz der Parteien}

Nach Salzburg brach eine Phase an, in der die politischen Parteien das Heft in die Hand nahmen. Die Linzer Länderkonferenz wurde zwar von den Ländern einberufen, und im Anschluss an Salzburg fanden weitere Besprechungen zwischen Staatssekretär Mayr und Ländervertretern statt. ${ }^{87}$ Der von Mayr ausgearbeitete Linzer Entwurf war jedoch sichtlich bemüht, den Parteien entgegenzukommen, namentlich den Deutschnationalen durch einen vom Volk gewählten Bundespräsidenten und den Sozialdemokraten durch eine Finanzhoheit des Bundes. ${ }^{88}$ Die Sozialdemokraten weigerten sich jedoch, ihn als überparteilichen Entwurf zu akzeptieren, und

\footnotetext{
${ }^{86}$ ERMACORA, Materialien 1, $193 \mathrm{f}$.

${ }^{87}$ VOLKER, Ausgleichsbestrebungen 145.

${ }^{88}$ Vgl. Art. 49 und Art. 10 Abs. 1 Z. 4 Linzer Entwurf, abgedruckt bei ERMACORA, Quellen 107 f, 119.
} 
legten mit einem Entwurf Dannebergs ${ }^{89}$ einen Gegenvorschlag vor, ohne ihn zu veröffentlichen. Auf der Konferenz selber, die vom 20. bis 23. April 1920 stattfand, hatten vollends die Parteien das Sagen. Eine Ursache mag darin gelegen sein, dass die Sozialdemokratische Arbeiterpartei infolge der Massendemonstrationen im März 1920 für die Vermögensabgabe verlorenen Einfluss zurück gewonnen hatte,,$^{90}$ ein zweiter Grund, dass innerhalb der Christlichsozialen die Wiener Richtung gegenüber den Landespolitikern wieder Boden gut machte. ${ }^{91}$

Die Linzer Beratungen brachten eine Annäherung der Standpunkte, die aber weiterhin durch fundamentalen Dissens über wichtige Zuständigkeiten und über Zuschnitt wie Funktion der Länderkammer überschattet war. ${ }^{92}$ Der Entwurf war den einen zu zentralistisch; die Sozialdemokraten, aber auch die Großdeutschen mahnten hingegen regelmäßig mehr Bundeskompetenzen ein, insbesondere in der Schule, bei den Landarbeitern, im Wasserrecht und im Elektrizitätswesen. Die Konferenz ging auseinander, ohne sich auf eine weitere Länderkonferenz zu verständigen. In der Folge entglitt den Ländern vollends die Initiative. ${ }^{93}$

Die Parteien füllten die Lücke und brachten teils vor, teils nach Zerbrechen der Koalition am 10. Juni $1920^{94}$ ihre Entwürfe in der Konstituierenden Nationalversammlung ein. ${ }^{95}$ Im Hinblick

\footnotetext{
${ }^{89}$ Zusammenfassung des Inhalts bei MATZKA, Sozialdemokratie und Verfassung $72 \mathrm{ff}$.

${ }^{90}$ HautmanN, Entstehungsbedingungen 45f.

${ }^{91}$ AltenstetTER, Föderalismus in Österreich 17.

92 Zusammenfassung und Bewertung bei ABLEITINGER, Grundlegung 172f.

${ }^{93}$ ERMACORA, Quellen 15; KOJA, Entstehung der Bundesverfassung 123.

${ }^{94}$ Dazu Goldinger, BINDER, Republik Österreich 82f.; BAUER, Österreichische Revolution 219f.; FUNDER, Vom Gestern ins Heute 500ff.; KUNSCHAK, Österreich 1918-1934, 62f.

${ }^{95}$ Den Anfang machten die Großdeutschen am 18. 5. 1920. Am 25. 6. folgten die Christlichsozialen, am 7. 7. die Sozialdemokraten.
}

auf die bundesstaatlichen Institutionen der Verfassung zeugen sie von beachtlicher Konvergenz. Alle Entwürfe enthielten bundesstaatliche Verfassungen, die eine Länderkammer vorsahen und der gleichen Kompetenzsystematik folgten. Der christlichsoziale und der sozialdemokratische Entwurf stimmten außerdem in vielen Detailfragen bis zu den Formulierungen überein. ${ }^{96}$ Das zeigt, dass die gemeinsame Arbeit an einem Regierungsentwurf nicht ohne Ergebnisse geblieben war.

\section{Juli 1920 bis Oktober 1920: Geburt in der Konstituierenden Nationalversammlung}

Die Einigung war also in greifbarer Nähe, und sie gelang im Laufe des Sommers und Frühherbsts 1920 in der Konstituierenden Nationalversammlung. ${ }^{97}$ Ein spezieller, mit der Verfassungsfrage betrauter Unterausschuss des Verfassungsausschusses konstituierte sich am 11. Juli 1920, mithin kurz nach Einbringung des Entwurfs der Sozialdemokraten.98 Den Sozialdemokarten, die nach dem Bruch der Koalition nicht mehr in der Regierung vertreten waren, fiel es nunmehr leichter, Kompromisse $\mathrm{zu}$ schließen, weil die Gefahr einer Spaltung der Partei gebannt erschien und die Basis nunmehr toleranter war. ${ }^{99}$

Die Beratungen begannen mit der Durchsicht der Kompetenzverteilung. ${ }^{100}$ Über die Systematik bestand seit Längerem Konsens, nicht aber

\footnotetext{
${ }^{96}$ Vgl. etwa das Erfordernisse einer Zweidrittelmehrheit für Beharrungsbeschlüsse im Landtag für den Fall, dass der Bundesrat einem Einspruch der Bundesregierung gegen das beschlossene Gesetz beigetreten war (vgl. Art. 27 Abs. 3 des 2. christlichsozialen und Art. 84 Abs. 3 des sozialdemokratischen Entwurfs, abgedruckt bei ERMACORA, Quellen 150, 171.

${ }^{97}$ Konziser Überblick über die Beratungen bei KOJA, Entstehung der Bundesverfassung 124ff.

98904 BlgKonstNV, eingebracht am 7. 7. 1920.

${ }^{99}$ HAUTMANN, Entstehungsbedingungen 48f.

${ }^{100} \mathrm{Vgl}$. ERMACORA, Quellen 268ff.
} 
über die Zuordnung politisch wichtiger Zuständigkeiten. Sowohl das Terrain als auch die Frontstellungen dieses Stellungskrieges blieben gleich: Die Sozialdemokraten traten regelmäßig für Bundeskompetenzen ein, die Christlichsozialen ebenso verlässlich für Länderkompetenzen. So manche Zuständigkeiten wurden zunächst hin, dann her und wieder zurück geschoben, und nicht selten gab in Pattsituationen die Position der Großdeutschen den Ausschlag. ${ }^{101}$ In der 6. Sitzung gelang eine wichtige Einigung über die Zusammensetzung des Bundesrates:102 Auf Vorschlag Kelsens verständigte man sich darauf, dem einwohnerreichsten Bundesland zwölf, jedem Land aber mindestens drei Abgeordnete zuzusprechen und im Übrigen am Grundsatz der verhältnismäßigen Vertretung festzuhalten. ${ }^{103}$ Auch die Dissense über den Einfluss des Bundesrats auf die Bundesgesetzgebung einerseits sowie über die Mitwirkung der Bundesregierung an der Landesgesetzgebung andrerseits wurden durch den Kompromiss aus dem Weg geräumt, die Ingerenzmöglichkeiten symmetrisch zu konstruieren: Bundesrat wie Bundesregierung sollten

\footnotetext{
${ }^{101}$ Vgl. die Nachweise über Änderungen und Umstellungen bei WALTER, Entstehung 45ff.

${ }^{102}$ Die Christlichsozialen waren zunächst für eine Vertretung jedes Landes durch drei Abgeordnete eingetreten (vgl. Art. 20 Abs. 1 des Entwurfs 231 BlgKonstNV = ERMACORA, Quellen 33f). Im 2. Entwurf schwenkten sie auf die Linie der Großdeutschen ein gestanden sie für Länder über 800.000 Einwohner vier Abgeordnete und für je weitere 400.000 Einwohner einen weiteren Abgeordneten zu (vgl. Art. 10 des Entwurfs 888 BlgKonstNV = ERMACORA, Quellen $142 \mathrm{f})$. Nicht übernommen ist das Modell der Großdeutschen, soweit nach ihm der Überschuss für volle 400.000 Einwohner gelten sollte, wenn er die Einwohnerzahl des kleinsten Landes überschritt (vgl. Art. 45 Abs. 2 des Entwurfs 842 BlgKonstNV= ERMACORA, Quellen 91). Die Sozialdemokraten traten für reine Proportionalität ein, wobei auf das kleinste Land ein Abgeordneter entfallen sollte (vgl. Art. 28 Abs. 1 Entwurf 904 BlgKonstNV = ERMACORA, Quellen 160). ${ }^{103}$ ERMACORA, Quellen 308.
}

Gesetze des föderalen Gegenübers durch Einspruch erschweren und verzögern, sie aber nicht verhindern können. ${ }^{104}$ Auf derselben Linie lag es, wenn von der Verankerung eines Vorranges des Bundesrechts abgesehen wurde, weil die lexposterior-Regel den auftretenden Konflikten bis zu ihrer Schlichtung durch den Verfassungsgerichtshof die Spitze zu nehmen vermöge. ${ }^{105}$ Entscheidungen gegen ein Austrittsrecht der Länder, gegen Neugliederungen der Länder aufgrund von Volksabstimmungen und gegen die Einräumung einer Kompetenz zum Abschluss von Staatsverträgen klärten weitere strittige Fragen. ${ }^{106}$

Sodann erhielt die mittelbare Bundesverwaltung ihre heutige Gestalt. Unter anderem wurde die Weisungsbefugnis der obersten Organe des Bundes explizit im Text verankert, ${ }^{107}$ schließlich in der 18. Sitzung, also buchstäblich fünf vor zwölf, der Landeshauptmann zur Drehscheibe dieser Institution gemacht. ${ }^{108}$ Die Auffassungsunterschiede über die Zuständigkeiten in Abgaben- und Schulangelegenheiten sowie der Dissens über die Demokratisierung der Verwaltung auf Bezirksebene konnten auch durch Verhandlungen der politischen Parteien nicht ausgeräumt werden. ${ }^{109}$ Diese Bereiche blieben daher ausgeklammert, und mit ihnen wurde auch die Rumpfverteilung, auf die man sich einigen konnte, mit einem Vorbehalt unterlegt. ${ }^{110}$

\footnotetext{
${ }^{104}$ M.w.N. SchefBECK, Entstehung des Bundesrates $337 f$.

${ }^{105}$ ERMACORA, Quellen 302f., 427, 553 = AB 991 BlgKonstNV 4.

${ }^{106}$ ERMACORA, Quellen 271, 334f. Einige dieser Anträge wurden im Plenum wiederholt.

${ }^{107}$ ERMACORA, Quellen 332.

108 ERMACORA, Quellen 489. Bis dahin war die Landesregierung vorgesehen (vgl. ebd. 404).

${ }^{109}$ ERMACORA, Quellen 424, 429ff., 500.

${ }^{110}$ Vgl. den Ausschussbericht, 992 BlgKonstNV 1 sowie den dort vorgeschlagenen § 42 Abs. 1 ÜG; vgl. auch die Erläuterungen Seipels im Plenum, StProtKonstNV 3378f.
} 
Die Debatte im Plenum begann am 29. September 1920 mit dem von Seipel erstatteten Bericht, der die bundesstaatlichen Institutionen sowie ihre Geburtswehen ins Zentrum rückte. ${ }^{111}$ Während Staatssekretär Mayr als Kabinettsvorsitzender ihren Kompromisscharakter betonte und sie verteidigte, ${ }^{112}$ ging Danneberg als Hauptredner der Sozialdemokraten zum Bundesstaat auf Distanz:113 Obschon seine Partei einen durchgängig auf dem Prinzip der Selbstverwaltung aufgebauten Einheitsstaat vorgezogen hätte, musste sie "[f]ür heute den tatsächlichen Machtverhältnissen Rechnung tragen".114 Die Arbeiterzeitung sekundierte und ätzte über die Detailversessenheit des Kompetenzkataloges. Die Abgrenzung der Zuständigkeiten sei bis zur Lächerlichkeit getrieben worden, man gewinne den Eindruck, „als würden sich da nicht Nations- und Staatsgenossen, sondern Feinde auseinandersetzen". ${ }^{115}$

Am 30. September 1920 setzte sich in der Plenarabstimmung über einige strittige Kompetenzfragen überwiegend die christlichsoziale Position durch. ${ }^{116}$ Nur mehr von symbolischer Bedeutung waren hingegen zwei Anträge, die beide auf eine Veränderung im Bestand der Länder abzielten. Nach dem Antrag Fink und Genossen sollte jedes Land innerhalb der ersten

\footnotetext{
111 Vgl. StProtKonstNV 3375ff.

112 StProtKonstNV 3383f.

113 Seine Rede sowie die Rede Leuthners wurden von der Partei als Broschüre gedruckt: KANE, Danneberg 124.

114 StProtKonstNV 3386.

115 Arbeiterzeitung vom 30. 9. 1920, 3.

116 Ihr Antrag, das Arbeitsrecht sowie die berufliche Vertretung der Landarbeiter in den Art. 12 zu überstellen, wurde nach einer Reassümierung widersprüchlicher Beschlüsse angenommen, während der großdeutsche Antrag auf Einfügung einer Bundeskompetenz für das Schul-, Erziehungs- und Volksbildungswesen in den Art. 10 die nötige Mehrheit verfehlte (vgl. 3346f. BlgKonstNV) und die Sozialdemokraten ihre Forderung nach Schaffung einer Bundeskompetenz für das Wasser- und das Elektrizitätswesen nicht in einen Antrag kleideten.
}

zehn Jahre des Bestandes der Bundesverfassung durch Volksabstimmung seinen Austritt aus dem Bund beschließen können, um sich einem anderen Staatswesen anzuschließen oder einen selbständigen Staat zu bilden. Nach dem Antrag Danneberg und Genossen sollten Gemeinden, deren Einwohner die Mehrheit in einem zusammenhängenden Landesgebiet bildeten, eine Volksabstimmung darüber verlangen können, ob dieses Gebiet einem angrenzenden Lande angegliedert oder ein neues Land bilden sollte. Beide Anträge verfehlten die nötige Mehrheit ${ }^{117}$. Sie zeigen aber, dass die Geburt bis zuletzt schwierig blieb. Nicht zu übersehen ist indes, dass es einen wichtigen Helfer gab. Noch im Plenum hat die Konstituierende Nationalversammlung einen umstrittenen Punkt dadurch gelöst, dass sie sich am Prinzip der Gleichrangigkeit und Ebenbürtigkeit von Bund und Ländern orientierte. ${ }^{118}$

\section{Oktober 1920 bis November 1920: Reaktionen der Länder auf die Bundesverfassung}

Meine Geschichte ist damit aber noch nicht zu Ende. Der Verabschiedung des B-VG in der KonstNV schließt sich eine Nachphase an, die bis zum 1. Dezember 1920, dem Tag der konstituierenden Sitzung des Bundesrates, ${ }^{119}$ währte.

Die Bundesverfassung 1920 machte ihr Inkrafttreten nicht von Willensbekundungen der Länder abhängig, die dem durch sie konstituierten Bundesstaat angehören sollten. ${ }^{120}$ Entgegen

\footnotetext{
117 Vgl. 3445 BlgKonstNV.

${ }^{118}$ Vgl. die Verständigung auf das Erfordernis eines qualifizierten Quorums (auch) für Beharrungsbeschlüsse des Landtages nach Einspruch der Bundesregierung (3451f. BlgKonstNV).

119 StProtBR 1. Sitzung vom 1. 12. 1920, $1 \mathrm{ff}$.

${ }^{120}$ Nach dem vom Verfassungsausschuss vorgeschlagenen Art. 2 Abs. 3 (991 BlgKonstNV) wäre dem Burgenland der Beitritt freigestellt gewesen. Erst das Plenum strich auf Antrag der Großdeutschen (vgl.
} 
politischer Beteuerungen waren die Länder auBerdem in die Beratungen der Konstituierenden Nationalversammlung nicht einbezogen worden. ${ }^{121}$ Gleichwohl akzeptierten alle Länder das Ergebnis und ordneten im November Vertreter in den Bundesrat ab. Nachdem sich aber vor allem die westlichen Länder als düpiert erachteten, ging das nicht ohne symbolische Gesten und nicht ohne Verwahrungen gegen das Prozedere der Verfassungsentstehung ab. ${ }^{122}$

Recht lakonisch reagierte Salzburg. Dort beschloss der Landtag am 25. November 1920 ein Gesetz über die Bildung des Bundesstaates Österreich. ${ }^{123}$ In dessen Art. I war angeordnet, dass das Land Salzburg mit den übrigen Ländern den Bundesstaat Österreich bildet, und der Art. II stellte klar, dass die Bildung „, auf Grundlage des von der Nationalversammlung in Wien am 1. Oktober 1920 beschlossenen Bundesverfassungsgesetzes" geschieht.

Andere Länder brachten ihren Unmut über das B-VG offener zum Ausdruck. In Kärnten verwahrte sich der Landtag durch Entschließung dagegen, dass die Bundesverfassung ohne überstimmenden Beschluss der Vertretungskörper der Gliedstaaten zustande gekommen sei. Er erklärte aber ,[m]it Rücksicht auf die schwierige Lage unseres Staates, die die Austragung grundsätzlicher verfassungsrechtlicher Streitigkeiten in diesem Augenblicke ausschließt", unter ausdrücklicher Wahrung seines Rechts-

3430f. BlgKonstNV) diesen Vorbehalt und fügte das Burgenland in die Aufzählung des Art. 2 Abs. 2 ein (vgl. 3444 BlgKonstNV).

${ }^{121}$ Erst der Entwurf des Unterausschusses bildet den Gegenstand von Verhandlungen, die aber nicht mit den Ländern selbst, sondern auf informeller Ebene mit Vertretern der Parteien in den Ländern geführt wurden. Zu ihrer Bedeutung vgl. den Bericht SEIPELS, StProtKonstNV 3377.

122 Eingehend dazu BERCHTOLD, Verfassungsgeschichte $267 \mathrm{ff}$.

${ }^{123}$ LGVBl. für das Land Salzburg 1920/168, kundgemacht am 30. 12. 1920. standspunkts die nachträgliche Zustimmung. ${ }^{124}$ Der ebenfalls mit Entschließung protestierende Tiroler Landtag konstatierte gar eine Verletzung der „dem Land kraft seines nach dem Umsturze wieder erlangten freien Selbstbestimmungsrechtes und dem Landtage auf Grund der geltenden Landesordnung zustehenden Rechte“. ${ }^{125}$

Zwei Länder gingen einen Schritt weiter und deklarierten ihre Mitgliedschaft zum Bundesstaat als eine vorläufige oder bedingte. Vorarlberg entsandte seine Abgeordneten in den Bundesrat „unter der feierlichen Verwahrung gegen die Annahme, dass dadurch in der provisorischen Zugehörigkeit des Landes und im Rechte des Landes auf freie Selbstbestimmung etwas geändert werde“ ${ }^{\prime 26}$ In Oberösterreich einigten sich alle Parteien, auch die Sozialdemokraten, auf eine gemeinsame Erklärung, die vom Landeshauptmann anlässlich der Wahl der Bundesratsabgeordneten im Landtag verlesen wurde. In ihr war festgehalten, dass sich Oberösterreich „als Bundesland mit den Ländern Burgenland, Kärnten, Niederösterreich (Niederösterreich Land und Wien), Salzburg, Steiermark, Tirol und Vorarlberg zum Bundesstaat Österreich" vereinige, ,jedoch nur insolange und soweit, als der Bundesstaat Österreich ein lebensfähiges Gebilde bleibt" ${ }^{\prime \prime}{ }^{127}$ Im Hinblick auf diese Kommentare und Vorbehalte war der erste Vorsitzende des Bundesrats, der Wiener Bürgermeister Reumann, in seiner Eröffnungsrede sichtlich um Versöhnung bemüht. ${ }^{128}$ Im neuen Verfassungswerk, das sich als Ausgleich zwischen zentralistischem Einheitsstaat und föderativer Staatsverbindung darstelle, hätten die Länder die Stellung „wahrer Gliedstaaten“ eines Bun-

\footnotetext{
${ }^{124}$ Veröffentlichungen über die Beratungen der Kärntner vorläufigen Landesversammlung in der Zeit vom 11. 11. 1918 bis 4. 7. 1921, Nr. 704, Sitzung 286.

${ }^{125}$ StProtLT 52. Sitzung vom 25. 11. 1920, 1374.

126 StProtLT 15. Sitzung vom 5. 11. 1920, 2.

127 StProtLT, 31. Sitzung vom 25. 11. 1920, 646.

128 StProtBR, 1. Sitzung vom 1. 12. 1920, 3.
} 
desstaates, die dem Bund vollkommen gleichberechtigt seien. Dieser Ausgleich lasse hoffen, dass eine Periode von Konflikten zwischen den Ländern und dem Bund zu Ende gegangen sei. Diese Hoffnung hat sich nur vordergründig erfüllt. Österreich ist zwar, sieht man von der nationalsozialistischen Ära und der Übergangsphase 1945/46 ab, ein Bundesstaat geblieben. Die im Laufe der Entwicklung immer kleinteiliger und unsystematischer gewordene Kompetenzverteilung, ${ }^{129}$ das Scheitern der Bundesstaatsreform zwischen 1992 und 1994, ${ }^{130}$ die vergeblichen Bemühungen im Österreich-Konvent zwischen 2003 und $2005^{131}$ und die unterschiedlich motivierte, im Ergebnis aber einhellige Ablehnung des von Expertengruppe Staats- und Verwaltungsreform ausgearbeiteten Vorschlags einer neuen Kompetenzverteilung ${ }^{132}$ machen aber gleichermaßen deutlich, dass die damals eröffnete Kluft zwischen Bund und Ländern bis heute nicht überbrückt ist. Die Bundesstaatlichkeit war neben den Grundrechten die größte Hürde, die auf dem Weg zu einer demokratischen Verfassung zu nehmen war, und sie hat sich - wiederum neben den Grundrechten - bei ihrer Weiterentwicklung wiederholt als Stolperstein erwiesen.

\section{Zusammenfassung}

Am Ende meines chronologischen Überblickes angelangt, fasse ich seine Ergebnisse kurz zusammen:

1. Dass Österreich als Bundesstaat eingerichtet wurde, haben die Länder erkämpft. Es ist nicht

\footnotetext{
${ }^{129}$ Unverändert gültige Problemdiagnose bei FUNK, Leistungsmängel $61 \mathrm{ff}$.

${ }^{130}$ Dokumentation in: Institut für Föderalismus, Materialien.

${ }^{131} \mathrm{Vgl}$. Österreich-Konvent (Hg.), Bericht des Österreich-Konvents Bd 1, 110ff.; Bd 2, $123 \mathrm{ff}$.

${ }^{132}$ Entwurf eines Bundesverfassungsgesetzes, mit dem das Bundes-Verfassungsgesetz geändert und ein Zweites Bundesverfassungsrechtsbereinigungsgesetz erlassen wird, 168/ME 23. GP.
}

leicht zu bestimmen, was sie dazu getrieben hat. An gliedstaatlichem Prestige waren sie jedenfalls nicht weniger interessiert als an Kompetenzen.

2. Zur Bundesstaatlichkeit gab es keine realistische Alternative. Wenn gleichwohl auf sozialdemokratischer Seite die eine oder andere Lanze für den Einheitsstaat gebrochen wurde, dann deshalb, um eine möglichst bundesfreundliche Ausgestaltung des Bundesstaates durchzusetzen oder um Bedürfnisse der eigenen Basis zu befriedigen.

3. Die Auffassungsunterschiede konnten vielfach durch Wahrung bundesstaatlicher Formen unter gleichzeitiger Preisgabe ihrer Funktionen überbrückt werden. Typisches Beispiel ist der Bundesrat, dessen Funktion darin besteht, da zu sein und im Übrigen keine Funktion zu haben.

4. Die Konstituierende Nationalversammlung hat die Parität von Bund und Ländern hochgehalten und sich bei der Ausgestaltung der bundesstaatlichen Institutionen an diesem Grundsatz orientiert. Das lag nahe, weil in der Monarchie Reichs- und Landesgesetze gleichen Rang hatten, und bot den Ländern eine gewisse Kompensation für die Dürftigkeit ihrer Kompetenzausstattung und ihres Einflusses im Bund. Wenn es einen genuin österreichischen Beitrag zur Ideengeschichte von Bundesstaatlichkeit und Föderalismus gibt, dann müssen wir ihn hierin suchen.

5. Schließlich und endlich: Das Verhältnis zwischen der Zentrale und den Ländern, im Oktober 1918 noch sehr harmonisch, hat sich die beiden nachfolgenden Jahre hindurch schrittweise verhärtet. Manche der damals geschlagenen Wunden sind bis heute nicht verheilt.

\section{Literatur:}

Alfred ABLEITINGER, Grundlegung der Verfassung, in: Erika WEINZIERL, Kurt SKALNIK (Hgg.), Österreich 1918-1938. Geschichte der Republik, Bd. 1 (GrazWien-Köln 1983) 147-194.

Ludwig Karl ADAMOVICH, Bernd-Christian FunK, Österreichisches Verfassungsrecht (Wien ${ }^{31985) . ~}$ 
Ludwig AdAmOvich (sen.), Hans SPANNER, Handbuch des österreichischen Verfassungsrechts (Wien 51957).

Christa AltEnSTETTER, Der Föderalismus in Österreich unter besonderer Berücksichtigung der politischen Verhältnisse von 1945-1966 (Heidelberg 1969).

Friedrich AUSTERLITZ, Staat und Länder, in: Der Kampf Jg. 12, Nr. 9 vom 31. 5. 1919, 329 ff.

Otto BAUER, Die österreichische Revolution (Wien 1923).

Klaus BERCHTOLD, Österreichische Parteiprogramme 1868-1966 (Wien 1967).

Klaus BERCHTOLD, Verfassungsgeschichte der Republik Österreich, Bd. 1: 1918-1933 (Wien 1998).

Wilhelm BRAUNEDER, Das Verhältnis Gesamtstaat Länder und die Entstehung der Republik Deutschösterreich, in: Alfred AbleITINGER (Hg.), Studien zur Zeitgeschichte der österreichischen Länder, Bd. 1: Demokratisierung und Verfassung in den Ländern 1918-1920 (St. Pölten 1983) 29-40.

Wilhelm BraUnEDER, Deutsch-Österreich 1918. Die Republik entsteht (Wien 2000).

Wilhelm BRAUNEDER, Karl Renners „Entwurf eines provisorischen Verfassung“: ein vorläufiger Bericht, in: Heinz MAYER u.a. (Hgg.), Staatsrecht in Theorie und Praxis. FS Walter (Wien 1991) 63-95.

Wilhelm BRAUNEDER, Österreichische Verfassungsgeschichte (Wien ${ }^{112009) . ~}$

Felix ERMACORA, Die Entstehung der Bundesverfassung 1920, Bd. 2: Dokumente der Staatskanzlei über allgemeine Fragen der Verfassungsreform (Wien 1989).

Felix ERMACORA, Die Entstehung der Bundesverfassung 1920, Bd. 4: Die Sammlung der Entwürfe zur Staats- bzw. Bundesverfassung (Wien 1990).

Felix ERMACORA, Materialien zur österreichischen Bundesverfassung, Bd. 1: Die Länderkonferenzen 1919/20 und die Verfassungsfrage (Wien 1989).

Felix ERMACORA, Österreichischer Föderalismus (=Schriftenreihe des Instituts für Föderalismusforschung 3, Wien 1976).

Felix ERMACORA, Quellen zum Österreichischen Verfassungsrecht (1920) (Wien 1967).

Felix ERMACORA, Christiane WIRTH, Die österreichische Bundesverfassung und Hans Kelsen (=Österreichische Schriftenreihe für Rechts- und Politikwissenschaft 4, Wien 1982).

Friedrich FundER, Vom Gestern ins Heute (Wien 31971).

Bernd-Christian FUNK, Leistungsmängel der bestehenden Kompetenzverteilung, in: Bundeskanzleramt (Hg.), Neuordnung der Kompetenzverteilung in Österreich (o.O, o.J. [Wien 1991]).
Walter GOLDINGER, Dieter BINDER, Geschichte der Republik Österreich 1918-1938 (Oldenburg 1992).

Ernst HANISCH, Der lange Schatten des Staates (Wien 1994).

Hans HaUtManN, Historisch-politische Entstehungsbedingungen des Bundes-Verfassungsgesetzes 1918-1920, in: Nikolaus DIMMEL, Alfred-Johannes Noll (Hgg.), Verfassung (Wien 1990) 19-63.

Ernst Carl Hellbling, Österreichische VerfassungsVerwaltungsrechtsgeschichte (Wien 21974).

Institut für Föderalismus (Hg.), Materialien zur Bundesstaatsreform (= Föderalismus-Dokumente 1, Innsbruck 1998).

Leon KANE, Robert Danneberg. Ein pragmatischer Idealist (= Schriftenreihe des Ludwig Boltzmann Instituts für Geschichte der Arbeiterbewegung) (Wien 1980).

Hans KelSEN (Hg.), Die Verfassungsgesetze der Republik Deutschösterreich, Bd. 1-3 (Wien-Leipzig 1919).

Hans Kelsen, Die Entwicklung des Staatsrechts in Österreich seit dem Jahre 1918, in: Gerhard ANSCHÜTZ, Richard THOMA (Hgg.), Handbuch des Deutschen Staatsrechts, Bd. 1 (Tübingen 1930) 147-165.

Hans KELSEN, Die Stellung der Länder in der künftigen Verfassung Deutschösterreichs, in: Zeitschrift für öffentliches Recht 1 (1919-20) 98-122.

Hans KELSEN, Österreichisches Staatsrecht (Tübingen 1923).

Friedrich F. G. KLEINWAECHTER, Von Schönbrunn nach St. Germain (Wien-Köln 1964).

Gottfried KÖFNER, Eine oder wieviele Revolutionen? Das Verhältnis zwischen Staat und Ländern in Deutschösterreich im Oktober und November 1918, in: Jahrbuch für Zeitgeschichte 1979 (1980) 131-167.

Friedrich KOJA, Die Entstehung der österreichischen Bundesverfassung, in: Österreich in Geschichte und Literatur 12 (1968) 121ff.

Leopold KUNSCHAK, Österreich 1918-1934 (Wien 1935).

Oskar LEHNER, Österreichische Verfassungs- und Verwaltungsgeschichte mit Grundzügen der Wirtschafts- und Sozialgeschichte (Linz ${ }^{4}$ 2007).

Manfred MATZKA, Sozialdemokratie und Verfassung, in: DERS (Hg.), Sozialdemokratie und Verfassung (Wien 1985) 9-155.

Adolf MerKL, Die Organisation der Gesetzgebung in der Deutschösterreichischen Republik, in: Zeitschrift für öffentliches Recht 1 (1919/20) 1-27.

Adolf MerKL, Die Verfassung der Republik Deutschösterreich (Wien 1919). 
Rudolf NECK, Sozialdemokratie, in: Erika WEINZIERL, Kurt SKALNIK (Hgg.), Österreich 1918-1938. Geschichte der Republik, Bd.1 (Graz-Wien-Köln 1983) 225-248.

Theo ÖHLINGER, Die Entstehung des Bundesstaates und ihre juristische Bedeutung, in: Salzburger Symposion zum Jubiläum 60 Jahre Bundesverfassung (Salzburg 1980) 41-49.

Theo ÖHLINGER, Rezension, in: ZNR 2 (1980) 113-115.

Österreich-Konvent (Hg.), Bericht des ÖsterreichKonvents, Bd. 1 und 2 (Wien 2005).

Peter PERNTHALER, Die Staatsgründungsakte der österreichischen Bundesländer (= Schriftenreihe des Instituts für Föderalismusforschung 14, Wien 1979).

Peter PERNTHALER, Österreichisches Bundesstaatsrecht (Wien 2004).

Karl RENNER, Das Selbstbestimmungsrecht der Nationen in besonderer Anwendung auf Österreich, Bd. 1: Nation und Staat (Wien ${ }^{21918) . ~}$

Günther SCHEFBECK, Zur Entstehung des Bundesrates, in: Herbert SCHAMBECK (Hg.), Bundesstaat und Bundesrat in Österreich (Wien 1997) 299-345.

Georg SchMiтZ, Die Verfassungsgespräche mit den österreichischen Ländern 1919/1920, in: ZNR (1979) 21-40.

Georg ScHMITZ, Die Vorentwürfe Hans Kelsens für die österreichische Bundesverfassung (= Schriftenreihe des Hans Kelsen-Instituts 6, Wien 1981).

Georg SchMitz, Karl Renners Briefe aus Saint Germain und ihre rechtspolitischen Folgen (= Schriftenreihe des Hans Kelsen-Instituts 16, Wien 1991).

Ignaz SEIPEL, Das Wesen des demokratischen Staates, in: Reichspost vom 20.11.1918, 1, wiederabgedruckt in: DERS., Der Kampf um die österreichische Verfassung (Wien 1930) 54-58.

Thomas SIMON, Der Föderalismus des Kaisertums Österreich nach 1860 und der Gedanke der Selbstverwaltung, in: Helmut NeuHAus (Hg.), Selbstverwaltung in der Geschichte Europas in Mittelalter und Neuzeit. Der Staat Beiheft 19 (Berlin 2010) 257-283.

Anton Staudinger, Christlichsoziale Partei, in: Erika WEINZIERL, Kurt SKALNIK (Hgg.), Österreich 19181938. Geschichte der Republik, Bd. 1 (Graz-WienKöln 1983) 249-276.
Anton Staudinger, Die Ereignisse in den Ländern Deutsch-Österreichs im Herbst 1918, in: Ludwig JEDLICKA (Hg.), Ende und Anfang Österreich 1918/19 (Salzburg 1969) 62-88.

Klaus Rüdiger VOLKER, Die Ausgleichsbestrebungen zwischen Zentralismus und Föderalismus bei den Länderkonferenzen 1918-1920 (phil. Diss. Salzburg 1977).

Robert WALTER, Die Entstehung des BundesVerfassungsgesetzes 1920 in der Konstitutierenden Nationalversammlung (= Schriftenreihe des Hans Kelsen-Instituts 9, Wien 1984).

Friedrich WALTER, Verfassung- und Verwaltungsrechtsgeschichte von 1500-1955 (Wien-Köln-Graz 1972).

Adam WANDRUSZKA, Das „nationale Lager“, in: Erika WEINZIERL, Kurt SKALNIK (Hgg.), Österreich 19181938. Geschichte der Republik, Bd. 1 (Graz-WienKöln 1983) 277-315.

Josef WERNDL, Kompetenzverteilung zwischen Bund und Ländern (= Schriftenreihe des Instituts für Föderalismusforschung 32, Wien 1984).

Leopold WERnER, Hans KLECATSKY, Das österreichische Bundesverfassungsrecht (Wien 1961).

Helmut WIDDER, Die Gesetzgebung, in: Herbert SCHAMBECK (Hg.), Das österreichische BundesVerfassungsgesetz und seine Entwicklung (Berlin 1980) 349-424.

\section{Abkürzungen:}

BlgKonstNV, Beilagen $\mathrm{zu}$ den stenographischen Protokollen der Konstituierenden Nationalversammlung

BlgProvNV Beilagen $\mathrm{zu}$ den stenographischen Protokollender Provisorischen Nationalversammlung

LGBl., LGVBl. Landesgesetzblatt, Landesgesetzund Verordnungsblatt

StProtBR Stenographische Protokolle des Bundesrates

StProtKonstNV Stenographische Protokolle der Konstituierenden Nationalversammelung

StProtLT Stenographische Protokolle des Landtages 